High Energy Processes and Phenomena in Astrophysics

IAU Symposium, Vol. 214, 2003

X. D. Li, V. Trimble, and Z. R. Wang, eds.

\title{
A Model for the Molecular Maser Disk in NGC4258
}

\author{
Natalia Babkovskaia and Juri Poutanen \\ Astronomy Division, P.O.Box 3000, FIN-90014 University of Oulu, \\ Finland
}

\begin{abstract}
A model of the $\mathrm{H}_{2} \mathrm{O}$ maser disk observed in the nucleus of the galaxy NGC4258 is presented. The hot-gas-cold-dust pumping mechanism based on the difference between gas and dust temperatures and special features of the ice spectrum is used. Conditions for the maser level inversion are calculated in the case of unsaturated maser. It is shown based on the observational data that the maser in NGC 4258 seems to be unsaturated. In that case the spectrum of the maser radiation at $\lambda=1.35$ cm including the central and high velocity components is calculated. The key parameters of the molecular maser disk are determined by comparing results of numerical simulations with the data.
\end{abstract}

\section{Introduction}

Considerable progress in elucidating the nature of active galactic nuclei has become evident in connection with a radiointerferometric study of the $\mathrm{H}_{2} \mathrm{O}$ maser emission from the nuclear region of the giant spiral galaxy NGC 4258. The line of sight lies virtually in the plane of the molecular gas-dust maser disk that is why we can observe the maser emission from the disk. In this study, an attempt is made to restore the structure of the accretion disk by comparing observational data with calculations of the intensity and spectrum of the maser emission.

Following Deguchi (1981) we consider the hot-gas-cold-dust maser pumping mechanism. This mechanism is based on the differences between gas and dust temperatures and the special features of the absorption coefficient of ice. The upper maser level is populated due to the absorption of $100 \mu \mathrm{m}$ and $85 \mu \mathrm{m}$ infrared photons corresponding to transitions $4_{14} \rightarrow 5_{05}$ and $5_{05} \rightarrow 6_{16}$ in ortho$\mathrm{H}_{2} \mathrm{O}$ molecule. The heat sink is realized due to the absorption of $45 \mu \mathrm{m}$ photons corresponding to the transition $5_{23} \rightarrow 4_{14}$ by cold ice dust which has a peak in the absorption coefficient at this wavelength.

\section{Model of Molecular Maser Disk in NGC 4258}

It was suggested in several papers that the maser in NGC 4258 is saturated (e.g. Collison and Watson 1995). According to our estimations the maser seems to be unsaturated. The fraction of the solid angle in which the medium is optically thick in the maser line is extremely small and the maser amplification takes place in a very narrow solid angle. In that case the inverse effect of maser emission on the signal level populations can be neglected and we can uncouple 
the population-balance and the radiative transfer equations. We first compute the population inversion of maser levels at every radius and then integrate the radiative transfer equation to obtain the radiation flux $F_{\nu}$ in the maser line of $\lambda=1.35 \mathrm{~cm}$. The high velocity features are produced by the spiral shear waves. Comparing the computed spectrum with the data allows us to reveal key parameters of the molecular maser disk. The results are presented in Fig. 1 and Table 1.

Table 1. Key parameters of the molecular maser torus in NGC4258

\begin{tabular}{ll|ll}
\hline Observational Data & & Model Parameters & \\
\hline Central mass & $3.6 \cdot 10^{7} M_{\odot}$ & Water concentration & $8 \cdot 10^{2} \mathrm{~cm}^{-3}$ \\
Distance & $6.4 \mathrm{Mpc}$ & Ice dust concentration & $4 \cdot 10^{-2} \mathrm{~cm}^{-3}$ \\
Maser spot area of & & Disk half thickness & $10^{14} \mathrm{~cm}$ \\
high velocity features & $3 \cdot 10^{32} \mathrm{~cm}^{2}$ & Hydrogen concentration & $10^{10} \mathrm{~cm}^{-3}$ \\
Inner radius & $0.13 \mathrm{pc}$ & Gas temperature & $400 \mathrm{~K}$ \\
Outer radius & $0.25 \mathrm{pc}$ & Ice temperature & $130 \mathrm{~K}$ \\
Temperature of & & Dust size & $10^{-5} \mathrm{~cm}$ \\
background radiation & $2 \cdot 10^{6} \mathrm{~K}$ & Maser ring width & $10^{15} \mathrm{~cm}$ \\
\hline
\end{tabular}
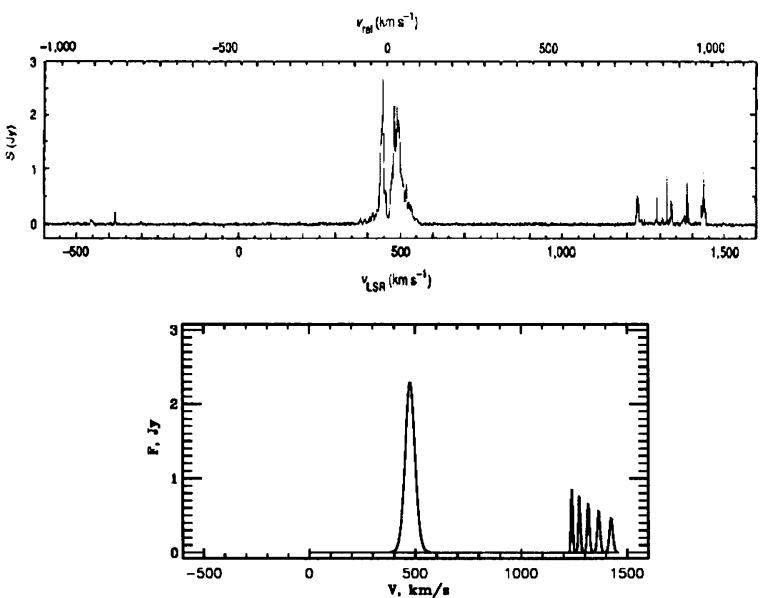

Figure 1. Spectrum of the $\mathrm{H}_{2} \mathrm{O}$ maser emission from NGC 4258: results of observations (top) and results of the numerical simulations (bottom).

Acknowledgments. This work was supported by the Centre for International Mobility, the Magnus Ehrnrooth Foundation, and the Finnish Graduate School for Astronomy and Space Physics.

\section{References}

Collison, A. \& Watson, W. 1995, ApJ, 452, L103

Deguchi, S. 1981, ApJ, 249, 145 